\title{
40 Gbit/S All-Optical Signal Regeneration with Soa In Mach-Zehnder Configuration
}

\author{
Gopal $^{1}$, Jimmy Gautam ${ }^{2}$, Ayushi Barthwal ${ }^{3}$ \\ ${ }^{I}$ (Electronics and communication department/ Galgotias University, Greater Noida, India, \\ ${ }^{2}$ (Electronics and communication department/ Amity University, Noida, India, \\ ${ }^{3}$ (Electronics and Communication Department/ Galgotias University, Greater Noida, India,
}

\begin{abstract}
In this paper, we propose an optical clock recovery (OCR) module using the fabry-perot filter (FPF) and semiconductor optical amplifier in Mach-zehnder configuration. An optical data stream of $40 \mathrm{Gbit/s}$ is input to the system and $40 \mathrm{GHz}$ optical clock signal is recovered. We have also presented method of $3 R$ optical signal regeneration by SOA in mach-zehnder (SOA-MZI) configuration by providing Re-amplification, Reshaping and Retiming using simple amplitude modulation.
\end{abstract}

Keywords: Fabry-perot filter, Optical clock recovery, SOA-MZI.

\section{INTRODUCTION}

All-optical signal transmission and processing are expected to enable the next generation of optical networks, by dramatically reducing costs, energy consumption, and increasing the network throughput. The increase of the per-channel bit rate usually leads to a reduction of the signal tolerance to optical impairments [1]. Such optical impairments are mostly originated from propagation and from signal processing. Propagation degrades the optical signal due to various distortion sources; such as: uncompensated chromatic dispersion, polarization mode dispersion, non-linear effects, or noise accumulation. Optical signal processing degrades the signals by tight optical filtering; crosstalk in photonic-cross connects; polarization dependent losses (PDL) of components; and by imperfect optical functionalities, like wavelength or format conversion.

Signal degradation in optical systems is typically overcome by adding regeneration stages at periodic intervals. The most common type of regeneration consists in detecting the optical signal, recovering it in the electrical domain and retransmitting it. However, this technique suffers from the general disadvantages of opticalelectronic-optical (OEO) signal processing. On the other hand, all-optical regenerators are expected to overcome the drawbacks of OEO converters and considered essential elements for fully-functional optical networks.

Regeneration can be divided in the three main functionalities: re-amplification, re-shaping, and retiming. The simplest functionality is re-amplification (or 1R), which consists in simple optical amplification. A device which also provides reshaping in addition to re-amplification is known as $2 \mathrm{R}$. Re-shaping consists in increasing the contrast between the two logical levels; which improves the required optical signal to noise ratio (OSNR) for a specific bit error rate (BER) level. Finally, $2 \mathrm{R}$ is combined with re-timing, to create a full 3R.

\section{OPERATION OF ALL-OPTICAL ClOCK RECOVERY USING SINGLE SOA}

All-optical SOA based schemes to extract optical clock include the use of a FPF [2] and a single SOA [3]. Alloptical clock recovery can be achieved through extracting the clock spectral components from optical signals with narrowband optical filtering. A Fabry-perot filter (FPF) with a free spectral range (FSR) matched to the signal bit-rate, and a large finesse (typically $>100$ ) is used. The optical spectrum of 40Gbit/s input signal consists of continuous spectral components from data modulation and line spectral components from the clock and its harmonics. When passing through the all-optical clock recovery module, the clock spectral components get transmitted, while the data modulation components get attenuated.

The FPF removes most of the data modulation and provides optical clock pulses matching to its FSR (bit-rate) frequency. Clock decays due to the presence of 0's that can be improved by using SOA-MZI at the input.

Actually, SOA [4] add 1's at the place of 0's.We have used the commercial software Optisystem from Optiwave, Inc., which allows the simulation at $40 \mathrm{Gbit} / \mathrm{s}$. The clock recovery setup is shown in Fig 1. It is composed of an FPF followed by an SOA which is used at the edge of non-linear region at $2.6 \mathrm{dBm}$. Fig. 2 depicts the spectrum of the input optical signal, consisting of a continuous spectrum and discrete lines; fabryperot filter that allows a filtering of the discrete lines of the spectrum if the FSR is close to the frequency modulation (bit rate). The filtered spectrum of the recovered Clock is also depicted. 


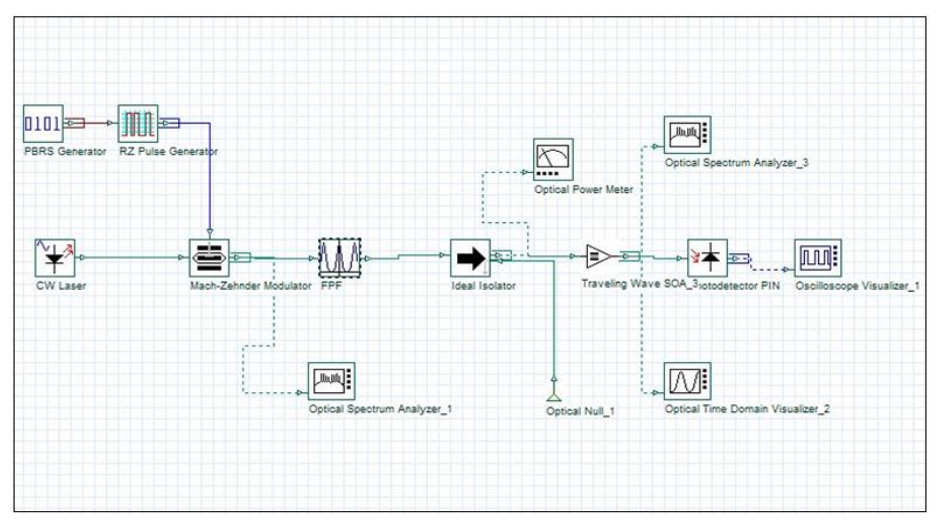

Fig. 1 Setup of clock recovery using single SOA

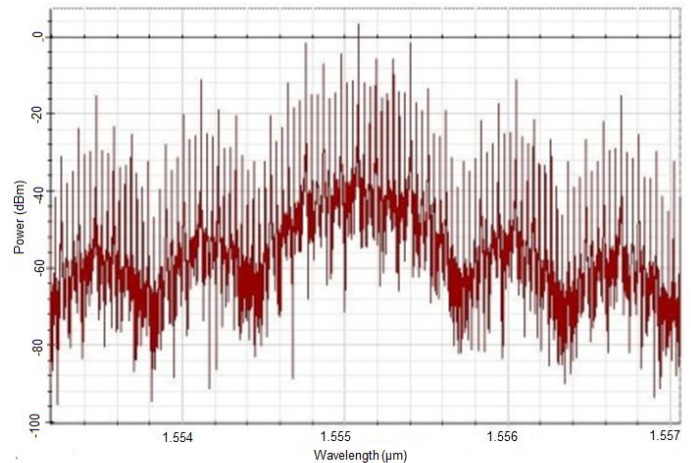

(a)

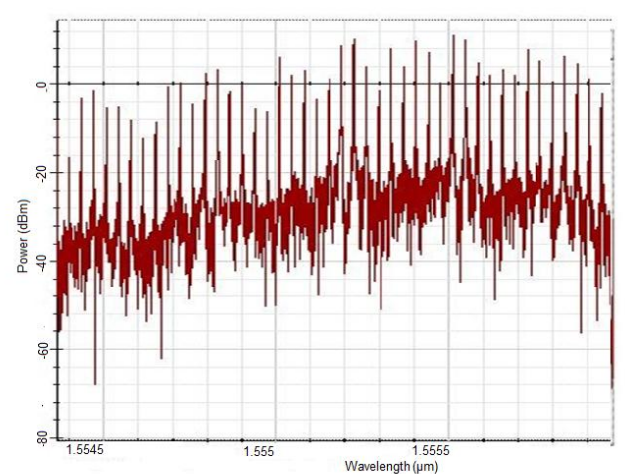

(b)

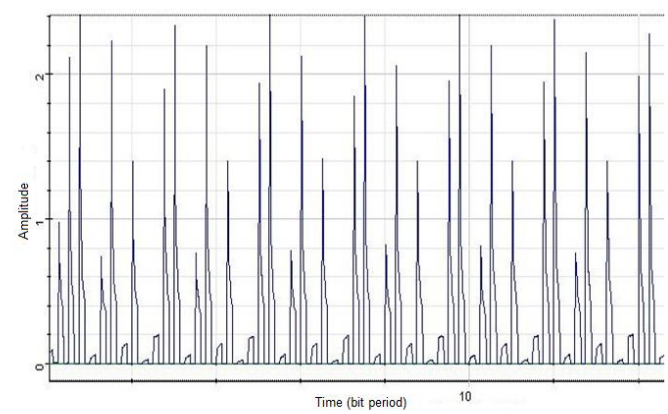

(c)

Fig. 2 (a) input data spectrum (b) clock spectrum(c) clock recovered

These basic spectra are obtained using our simulation results. It appears that the quality of the Recovered Clock depends intrinsically on filter characteristics, and particularly on the finesse value. The filter being not ideal, a residual part of the continuous spectrum is transmitted and present in the output spectrum The number of bits per sequence was varied from $2^{7}-1$ to $2^{13}-1$ (i.e., $127-8191$ bits/sequence) at a bit rate of $40 \mathrm{Gbit} / \mathrm{s}$. The physical parameters used for the simulation are: the SOA input power equal to $2.6 \mathrm{dBm}$, the SOA current drive equal to $300 \mathrm{~mA}$, the SOA length equal to $500 \mu \mathrm{m}$, the effective area equal to $2.4 \times 10^{-13} \mathrm{~m}^{2}$ ,confinement factor equal to 0.3 .

\section{OPERATION OF ALL-OPTICAL ClOCK RECOVERY USING SOA-MZI}

One simple method to regenerate the signal is presented in Fig 3. The clock-recovery consists in a first stage, where the information signal is filtered using a Fabry-Perot filter (FPF), which extracts the clock components of the signal. The power of the pulses at the FPF filter output fades exponentially for consecutive ' 0 ' of the input signal. Therefore, a SOA-MZI [5] wavelength conversion stage is used to equalize the power of the optical pulses. Power input to the SOA-MZI is $2.2 \mathrm{dBm}$ in non-linear region. Another wavelength conversion stage could be used before FPF that can improve clock and equalize signal to FPF. Retiming is done by using amplitude modulation between the clock recovered and transmitted data signal. Other physical parameters of SOA remain the same. 


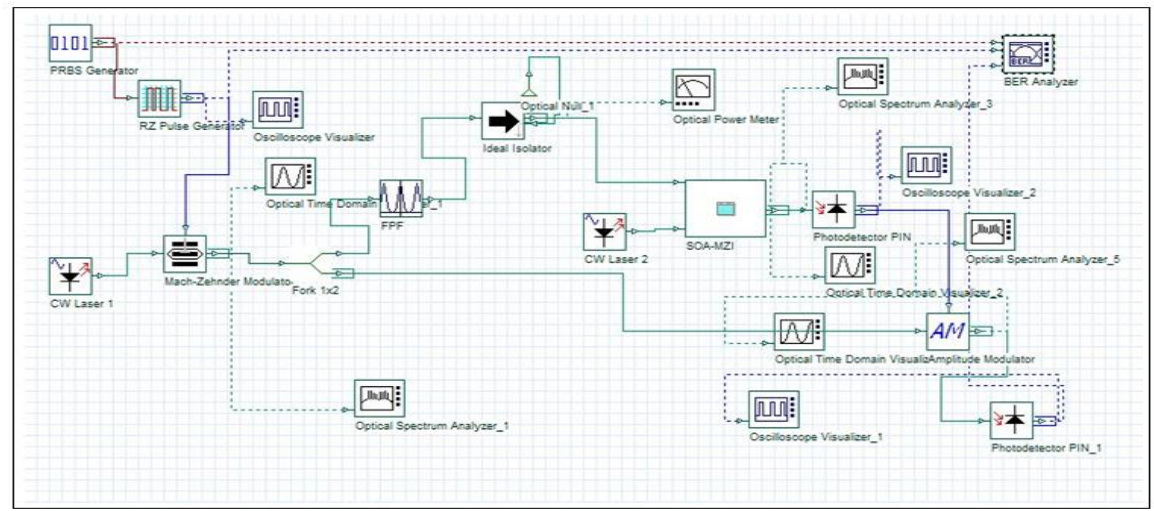

Fig. 3 Operation of All-optical wavelength regeneration using SOA-MZI
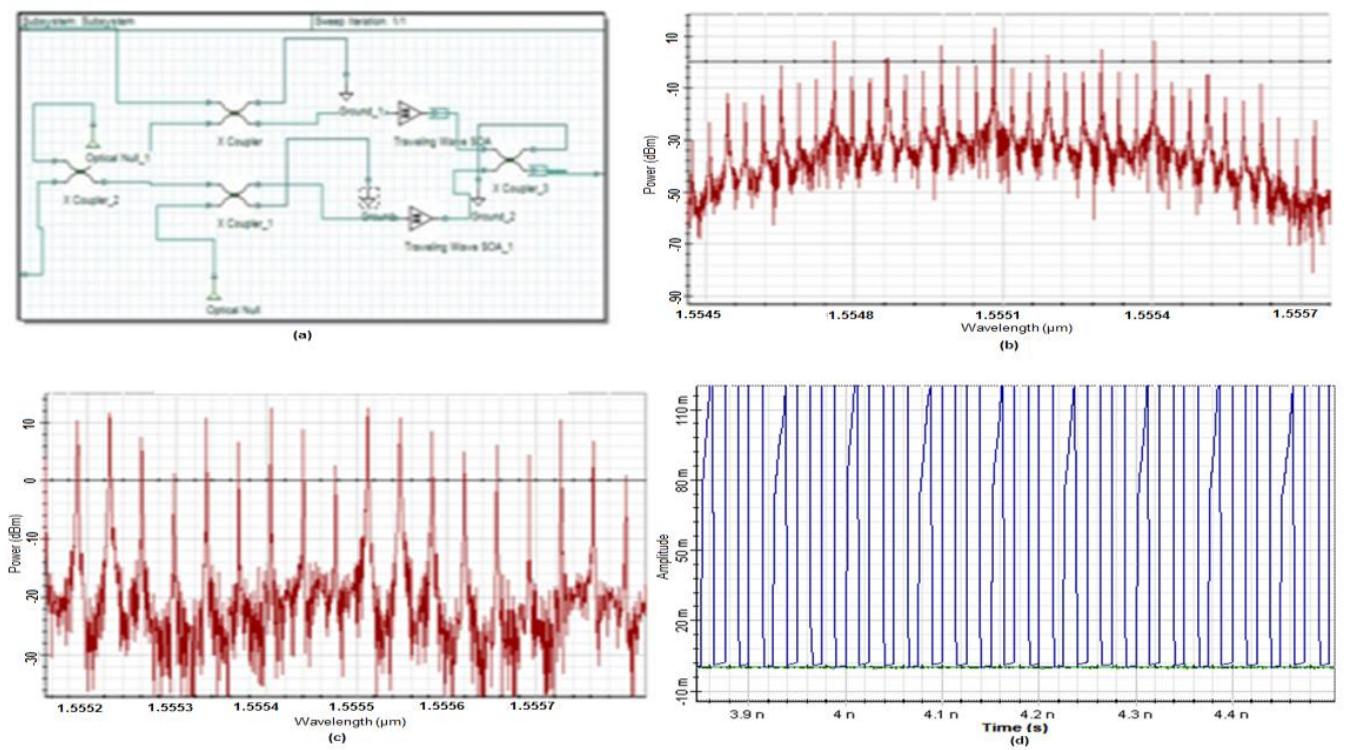

Fig. 4 (a) SOA-MZI (b) input data spectrum (c) clock spectrum (d) clock recovered

On comparing, The Results of clock recovered from Fig. 2(c) and Fig.4 (d), we can observe that clock recovered by single SOA decays more than that of using SOA-MZI. The amplitude varies slowly and at large sequences of 0's, Amplitude of clock is high than single SOA. The BER of single SOA varies between $10^{-13}$ to $10^{-18}$ while BER of SOA-MZI varies between $10^{-28}$ to $10^{-32}$ on the basis of presence of 1's and 0 's in the PRBS generated sequences.

\section{CONCLUSION}

We have demonstrated and simulated $40 \mathrm{Gbit} / \mathrm{s}$ all-optical wavelength regeneration by SOA's in a Mach-zehnder configuration using OptiSystem. We have presented wavelength regeneration at $40 \mathrm{Gbit} / \mathrm{s}$ with BER less than $10^{-30}$. Working together with a FPF-based all-optical clock recovery module, this technique achieved modulation-format-independent all-optical clock recovery with a simple and straight-forward configuration. When the data modulation contents long sequences of repeated zeros, the recovered clock amplitude can decay due to pattern-dependence. By using SOA in Mach-zehnder configuration, we can improve recovered clock. The simulation results showed that the SOA-MZI could sharpen the clock components in the input signal and reduce both the timing jitter and amplitude fluctuations on the recovered clocks.

\section{REFERENCES}

[1] G. Keiser, Optical Fiber Communications (Boston (USA): Mc GRAW-HILL, 2000) 166 - 171.

[2] J. Stone and L.W.Stulz, Pigtailed High-Finesse Tunable Fibre Fabry-Perot Interferometers with Large, Medium and Small Free Spectral Ranges, Electronic Letters, 23(15), 1987, 781 - 783.

[3] V. Roncin, S. Lobo, M. Ngo, L. Bramerie, A. O’Hare, M. Joindot, and J. C. Simon, Patterning Effects in All-Optical Clock Recovery: Novel Analysis Using a Clock Remodulation, IEEE J. Of selected topics in quantum electronics, 16(5), 2010.

[4] F. Ohman, S. Bischoff, B. Tromborg, J. Mork, Semiconductor devices for all-optical regeneration, proc. ICTON, 2, 2003 , 41-46.

[5] M. Spyropoulou, N. Pleros, and A. Miliou, SOA-MZI-Based Nonlinear Optical Signal Processing: A Frequency Domain Transfer Function for Wavelength Conversion, Clock Recovery, and Packet Envelope Detection, IEEE J. of Quantum Electronics, 47, 2011. 